\title{
On the Complementary Wave Interpretation of the Dirac Equation
}

\author{
D. L. Bulathsinghala, K. A. I. L. Wijewardena Gamalath* \\ Department of Physics, University of Colombo, Colombo 3, Sri Lanka \\ *E-mail address: imalie@phys.cmb.ac.lk
}

\begin{abstract}
The Dirac equation consistent with the principles of quantum mechanics and the special theory of relativity, introduces a set of matrices combined with the wave function of a particle in motion to give rise to the relativistic energy-momentum relation. In this paper a new hypothesis, the wave function of a particle in motion is associated with a pair of complementary waves is proposed. This hypothesis gives rise to the same relativistic energy-momentum relation and achieves results identical to those of Dirac. Additionally, both the energy-time and momentum-position uncertainty relations are derived from the complementary wave interpretation. How the complementary wave interpretation of the Dirac equation is related to the time-arrow and the four-vectors are also presented.
\end{abstract}

Keywords: The Dirac equation; energy-momentum equation; complementary waves; uncertainty relation; time-arrow; four-vector

\section{INTRODUCTION}

Since the introduction of the quantum mechanics in 1927, there have been two difficult problems associated with the physical theory. The first question was, and still is, whether one should be satisfied with the probabilistic interpretation of quantum mechanics. The second question is whether the probabilistic interpretation which is inherent in the present form of quantum mechanics can accommodate special relativity. As it stands, this is understood to be one of the biggest inconsistencies between relativity and quantum mechanics. Relativistic wave-equations describe particles as waves, or more generally quantum energy fields which fluctuate like waves. The failure of classical mechanics to explain atomic phenomena gave rise to the need for a new mechanics, later termed quantum mechanics. The quantum revolution started in 1900 when Max Planck showed [1] how the black-body radiation problem can be resolved, by assuming the radiation energy coming out of a black-body was quantized. Einstein was then instrumental in explaining the photoelectric effect [2], stating that light energy comes in the form of discrete quanta. Later De-Broglie, Bohr, Schrodinger, Heisenberg, Pauli and Dirac were instrumental in developing the mathematical formulation of quantum mechanics. 
In non-relativistic quantum mechanics the correspondence principle dictates that the momentum operator is associated with the spatial gradient, and the energy operator with the time derivative. Since four momentum $P^{\mu}=(E / c, \boldsymbol{p})$ transforms like a 4-vector under Lorentz transformations, the operator, $\hat{P}^{\mu}=i \hbar \partial^{\mu}(\mu=0,1,2,3)$ is relativistically covariant. Non-relativistic Schrödinger equation is obtained by quantizing the classical Hamiltonian

$$
i \hbar \frac{\partial \psi}{\partial t}=-\frac{\hbar^{2}}{2 m} \nabla^{2} \psi+V \psi
$$

Schrödinger equation cannot be relativistically correct, as it does not contain space and time derivatives of the same order. Therefore it is impossible to "mix" the space-time coordinates in the manner required by relativity, and end up with the same equation as one started with. Historically, the first attempt to construct a relativistic version of the Schrödinger equation began by applying the familiar quantization rules to the relativistic energy-momentum invariant $P^{\mu} P_{\mu}$ :

$$
P^{\mu} P_{\mu}=E^{2} / c^{2}-p^{2}=m^{2} c^{2}
$$

leading to a quadratic relation known as the Klein-Gordon equation:

$$
-\hbar^{2} \partial_{t}^{2} \psi=\left(-\hbar^{2} c^{2} \nabla^{2}+m^{2} c^{4}\right) \psi
$$

As the Klein-Gordon equation is quadratic in nature, it predicts negative energies and negative probabilities. Motivated by Schrodinger's wave equation and Heisenberg's matrix mechanics, it was Dirac who first sought to formulate a relativistic wave equation applicable for particles at relativistic speeds, such as electrons. As it turned out, any solution to the Dirac equation was automatically a solution to the Klein-Gordon equation, but the converse was not true. Starting with Schrodinger equation, Dirac introduced a set of non-commuting matrices $\alpha^{j}(j=1,2,3)$ and $\beta$ and formulated the Dirac equation [3]:

$$
\left(c \alpha^{j} p_{j}+m c^{2} \beta\right) \psi=\left(-i \hbar c \alpha^{j} \partial_{j}+m c^{2} \beta\right) \psi=E \psi
$$

where $\beta^{2}=I_{4}$ and $\left\{\alpha^{i}, \alpha^{j}\right\}=2 \delta_{i j} ; \quad\left\{\alpha^{i}, \beta\right\}=0 \forall i$.

Soon after Dirac formulated his relativistic wave equation in 1928, he attempted to explain the unavoidable negative-energy solution for the relativistic electron [4] but Robert Oppenheimer strongly argued against the proton being the negative solution to the Dirac equation. But Dirac then published a paper in 1931 [5] deducing the existence of an "antielectron" later termed as the positron and in 1932 Carl Anderson discovered the predicted positrons [6]. The Dirac equation is now known to apply for all massive spin half fermions in relativistic limits.

As the Dirac equation was not sufficient to incorporate particles with arbitrary spin, other equations were proposed such as the Majorana equation [7], Rarita-Schwinger equation [8], Proca-equation [9], Bhabha-equation [10] and Bargmann-Wigner equation [11]. A 
detailed account of their work is found in a paper entitled: "Searching for an Equation: Dirac, Majorana and the Others" [12]. Since then, various theorists did further research in relativistic Hamiltonians for particles with higher-spins [13-15]. Although the Dirac equation is considered as the generally accepted relativistic wave interpretation for relativistic particles, there were few other interpretations to the Dirac equation without using spinors. In 1967, Hestenes proposed an alternative proposal based on the space-time Clifford algebra [16], which uses four-vectors forming the basis of the space-time, instead of four- $\gamma$ matrices [17].

In this paper, a new hypothesis that a particle in motion is associated with a pair of complementary waves is presented. This concept gives rise to the same relativistic energymomentum relation and achieves results identical to those of Dirac. In this representation, the components of the relativistic energy-momentum relation are observed energy quantities arising from the proposed complementary waves. Taking the inner product of the proposed complementary wave interpretation of the Dirac equation, the conventional four vectors are obtained. An exact solution of the complementary wave interpretation is presented and the energy-time and momentum-position uncertainty relations were derived. A relationship between the complementary wave interpretation of the Dirac equation and the time-arrow is obtained, dictating how events unfold in the real world. The present paper is an effort to represent the Dirac equation in accordance with both the energy-time and momentumposition uncertainty relations and thereby to give a simplified description of elementary particles, consistent with both the principles of quantum mechanics and the theory of special relativity.

\section{COMPLEMENTARY WAVE INTERPRITATION OF THE DIRAC EQUATION}

The Schrodinger equation for a particle in motion is associated with a singlecomponent wave function. Therefore, to make it comply with the relativistic energymomentum relation, Dirac introduced a set of matrices matrices $\alpha^{j}$ and $\beta$ and formulated the Dirac equation. Here, instead of combining the matrices $\alpha^{j}$ and $\beta$ to a single normalized wave function $\psi(\boldsymbol{r}, t)$, the matrices $\alpha^{j}$ and $\beta$ are combined to a pair of normalized complementary waves $\psi_{0}$ and $\psi_{1}$ such that:

$$
E \psi=\beta m c^{2} \psi_{0}+\alpha^{j} p_{j} c \psi_{1} \quad(j=1,2,3)
$$

By taking the inner-product of equation 5:

$$
\langle E \psi \mid E \psi\rangle=\left\langle\beta m c^{2} \psi_{0}+\alpha^{j} p_{j} c \psi_{1} \mid \beta m c^{2} \psi_{0}+\alpha^{j} p_{j} c \psi_{1}\right\rangle
$$

we obtain,

$E^{2}\langle\psi \mid \psi\rangle=m^{2} c^{4} \beta^{2}\left\langle\psi_{0} \mid \psi_{0}\right\rangle+\left(\alpha^{j} p_{j}\right)^{2} c^{2}\left\langle\psi_{1} \mid \psi_{1}\right\rangle+m c^{3} p_{j}\left(\beta \alpha^{j}\left\langle\psi_{0} \mid \psi_{1}\right\rangle+\alpha^{j} \beta\left\langle\psi_{1} \mid \psi_{0}\right\rangle\right)+\frac{1}{2}\left(\alpha^{i} \alpha^{j}+\alpha^{j} \alpha^{i}\right) p_{i} p_{j} c^{2}\left\langle\psi_{1} \mid \psi_{1}\right\rangle$.

where $i$ and $j$ are distinct. Using the anti-commutative properties of the Dirac matrices given in equation 4 and the normalized properties of the wave functions, equation 7 reads, 


$$
E^{2}=m^{2} c^{4}+p^{2} c^{2}+m c^{3} \beta \alpha^{j} p_{j}\left(\left\langle\psi_{0} \mid \psi_{1}\right\rangle-\left\langle\psi_{1} \mid \psi_{0}\right\rangle\right)
$$

As $\psi_{0}$ and $\psi_{1}$ are a complementary wave pair, $\int_{R} \psi_{0}^{*} \psi_{1} d^{3} r \neq \int_{R} \psi_{1}^{*} \psi_{0} d^{3} r$ :

$$
\left\langle\psi_{0} \mid \psi_{1}\right\rangle \neq\left\langle\psi_{1} \mid \psi_{0}\right\rangle \text {. }
$$

Therefore, in order to obtain the energy-momentum relation, equation 8 has to be integrated and averaged over the periodic temporal interval $T$ :

$$
\frac{1}{T} \int_{0}^{T} E^{2} d t=\frac{1}{T} \int_{0}^{T}\left(m^{2} c^{4}+p^{2} c^{2}\right) d t+\frac{1}{T} \int_{0}^{T} m c^{3} \beta \alpha^{j} p_{j}\left(\left\langle\psi_{0} \mid \psi_{1}\right\rangle-\left\langle\psi_{1} \mid \psi_{0}\right\rangle\right) d t
$$

where the periodic temporal interval $T$ representing the orthogonal periodic interval between the two complementary waves, satisfy the orthogonal condition:

$$
\int_{0}^{T} d t\left\langle\psi_{0} \mid \psi_{1}\right\rangle=\int_{0}^{T} d t \int_{-\infty}^{+\infty} d^{3} r \psi_{0}^{*} \psi_{1}=0=\int_{0}^{T} d t\left\langle\psi_{1} \mid \psi_{0}\right\rangle
$$

then, equation 10 leads to,

$$
E^{2}=p^{2} c^{2}+m^{2} c^{4}
$$

As the relativistic energy-momentum relation can be obtained by associating a pair of complementary waves $\psi_{0}$ and $\psi_{1}$ to a particle in motion, a complementary wave interpretation to the Dirac equation is obtained.

\section{FOUR VECTORS ASSOCIATED WITH COMPLEMENTARY WAVES}

In the theory of relativity, a four-vector $A^{\mu}(\mu=0,1,2,3)$ is a four dimensional vector in Minkowski spacetime, defined as a quantity which transform under Lorentz transformation in the same way as coordinates of a point $\left(x_{0}, x_{1}, x_{2}, x_{3}\right)$ giving rise to a scalar product $A^{\mu} A_{\mu}$ which is invariant under Lorentz transformation. However, in the complementary wave interpretation, the momentum-energy and the rest-energy components are associated with a pair of complementary waves. Thereby introducing total energy as $\gamma m c^{2}$, where the Lorentz factor $\gamma=\left(1-u^{2} / c^{2}\right)$ is given in terms of the velocity of the particle $\boldsymbol{u}$, equation 5 reads:

$$
\begin{gathered}
\left(\gamma m c^{2}\right) \psi=\beta m c^{2} \psi_{0}+\alpha^{j} p_{j} c \psi_{1}=m c\left(\beta c \psi_{0}+\alpha^{j} \gamma u_{j} \psi_{1}\right) \\
(\gamma c) \psi=\left(\beta c \psi_{0}+\alpha^{j} \gamma u_{j} \psi_{1}\right) .
\end{gathered}
$$


Taking the inner-product of equation 14 and averaging over a periodic temporal interval $T$, the properties of Dirac matrices give:

$$
\frac{1}{T} \int_{0}^{T}\langle\gamma c \psi \mid \gamma c \psi\rangle d t=\frac{1}{T} \int_{0}^{T}\left\langle\beta c \psi_{0}+\gamma \alpha^{j} u_{j} \psi_{1} \mid \beta c \psi_{0}+\gamma \alpha^{j} u_{j} \psi_{1}\right\rangle d t \Rightarrow c^{2} \gamma^{2}=c^{2}+\gamma^{2} u^{2}
$$

Since the proper time $d \tau=d t / \gamma$, equation 15:

$$
\begin{gathered}
(c d t)^{2}=(c d \tau)^{2}+(u d t)^{2} \Rightarrow(c d \tau)^{2}=(c d t)^{2}-d x^{2}-d y^{2}-d z^{2}=d S^{2} \\
d S^{2}=d X^{\mu} d X_{\mu}=\eta_{\mu \nu} d X^{\mu} d X^{\nu} \Rightarrow d X^{\mu}=(c d t, d x, d y, d z)
\end{gathered}
$$

where the diagonal terms of the metric tensor $\eta_{\mu \nu}$ are $(1,-1,-1,-1)$. This leads to the conventional four-position vector $X^{\mu}=(c t, \boldsymbol{r})=\left(x_{0}, x_{1}, x_{2}, x_{3}\right)$ in Minkowskian spacetime. Similarly staring from the complementary interpretation of the Dirac equation four velocity $U^{\mu}=d X^{\mu} / d \tau=\gamma(c, \boldsymbol{u})$, four momentum $P^{\mu}=m U^{\mu}=(E / c, \boldsymbol{p})$ and using De-Broglie's matter-wave hypothesis[18], four wave vector $K^{\mu}=P^{\mu} / \hbar=(\omega / c, k)$ can be obtained.

As $\psi_{0}$ and $\psi_{1}$ are a complementary wave pair, equation 13 suggests that the physical quantity $\gamma m c^{2}$ or its derivatives in four-vector notation $c t, \gamma c, E / c, \omega / c$ of a particle in motion are associated with two complementary states and that any two physical quantities associated with the complementary states cannot be measured with arbitrary precision. For instance, one cannot know both the position associated with the rest-energy and the momentum associated with the momentum-energy of a particle in motion, simultaneously with absolute precision. This we identify as the Heisenberg's uncertainty principle. Further, as the total energy $\gamma m c^{2}$ of a particle in motion is associated with two complementary states: i.e. rest-state associated with the rest-energy and momentum-state associated with the momentum-energy, the complementary wave interpretation suggests that the particle motion is a quantized phenomena.

\section{EXACT SOLUTION OF THE COMPLEMENTARY WAVE INTERPRITATION}

The inner product of four momentum $P^{\mu}$ and four position vector $X^{\mu}$,

$$
P^{\mu} X_{\mu}=(E / c, \boldsymbol{p})(c t,-\boldsymbol{r})=E t-\boldsymbol{p} \cdot \boldsymbol{r}=m c^{2} \tau
$$

Applying $E=\hbar \omega$, and $p=\hbar k$ :

$$
\omega_{0} \tau=\omega t-\boldsymbol{k} \cdot \boldsymbol{r}
$$


Considering a plane wave solution which combines, $e^{-i \omega_{0} \tau}$ and $e^{-i(\omega t-\boldsymbol{k} \cdot \boldsymbol{r})}$ and applying the four dimensional derivative operator, $\frac{1}{c} \frac{d}{d \tau}=\gamma^{\mu} \partial_{\mu}$ where $\partial_{\mu}=\left(\frac{1}{c} \frac{\partial}{\partial t}, \nabla\right)$,

$$
\frac{1}{c} \frac{d}{d \tau}\left(e^{-i \omega_{0} \tau}\right)=\gamma^{\mu} \partial_{\mu}\left(e^{-i(\omega t-\boldsymbol{k} \cdot \boldsymbol{r})}\right) \Rightarrow \frac{-i \omega_{0}}{c} e^{-i \omega_{0} \tau}=-\frac{i \gamma^{0}}{c} \omega e^{-i(\omega t-\boldsymbol{k} \cdot \boldsymbol{r})}+i \gamma^{j} k_{j} e^{-i(\omega t-\boldsymbol{k} \cdot \boldsymbol{r})}
$$

Since $\gamma^{0}=\beta, \gamma^{j}=\beta \alpha^{j}$ multiplying by $\beta \hbar c$, the above equation can be rearranged to read,

$$
E e^{-i(\omega t-\boldsymbol{k} \cdot \boldsymbol{r})}=\beta m c^{2} e^{-i \omega_{0} \tau}+\alpha^{j} p_{j} c e^{-i(\omega t-\boldsymbol{k} \cdot \boldsymbol{r})} \Rightarrow E \psi=\beta m c^{2} \psi_{0}+\alpha^{j} p_{j} c \psi_{1} .
$$

Equation 20 leads to Dirac equation, since with $\psi_{0}=\psi_{1}=\psi$ from equation 18.

$$
E \psi=\beta m c^{2} \psi+\alpha^{j} p_{j} c \psi \quad(j=1,2,3) .
$$

However, there are eight possible ways, the two waves $e^{-i \omega_{0} \tau}$ and $e^{-i(\omega t-\boldsymbol{k} \cdot \boldsymbol{r})}$ can be combined with the four derivative operator i.e.

$$
\begin{aligned}
& \frac{1}{c} \frac{d}{d \tau}\left(e^{-i \omega_{0} \tau}\right)=\gamma^{\mu} \partial_{\mu}\left( \pm e^{-i(\omega t-\boldsymbol{k} \cdot \boldsymbol{r})}\right) ; \quad \frac{1}{c} \frac{d}{d \tau}\left(e^{i \omega_{0} \tau}\right)=\gamma^{\mu} \partial_{\mu}\left( \pm e^{-i(\omega t-\boldsymbol{k} \cdot \boldsymbol{r})}\right) \\
& \frac{1}{c} \frac{d}{d \tau}\left(e^{-i \omega_{0} \tau}\right)=\gamma^{\mu} \partial_{\mu}\left( \pm i e^{-i(\omega t-\boldsymbol{k} \cdot \boldsymbol{r})}\right) ; \quad \frac{1}{c} \frac{d}{d \tau}\left(e^{i \omega_{0} \tau}\right)=\gamma^{\mu} \partial_{\mu}\left( \pm i e^{-i(\omega t-\boldsymbol{k} \cdot \boldsymbol{r})}\right)
\end{aligned}
$$

But only the waves given in equations 23 are orthogonal and out of them only the two sets of waves given by

$$
\frac{1}{c} \frac{d}{d \tau}\left(e^{i \omega_{0} \tau}\right)=\gamma^{\mu} \partial_{\mu}\left( \pm i e^{-i(\omega t-\boldsymbol{k} \cdot \boldsymbol{r})}\right)
$$

satisfy the conditions given in equation 9 and 11 . These two solutions can be further generalized as,

$$
\psi_{0}= \pm i \psi_{1}^{*}
$$

where the two waves in each solution form a complementary wave-pair. From equation 24, the following wave-equations can be obtained:

$$
E e^{-i(\omega t-\boldsymbol{k} \cdot \boldsymbol{r})}= \pm i \beta m c^{2} e^{i \omega_{0} \tau}+\alpha^{j} p_{j} c e^{-i(\omega t-\boldsymbol{k} \cdot \boldsymbol{r})}
$$

As both these equations give the energy-momentum relation, equation 26 represents the generalized complementary wave interpretation. From the plane wave solutions given in equation 25 , the following inner products can be obtained: 


$$
\begin{aligned}
& \left\langle\psi_{0} \mid \psi_{1}\right\rangle-\left\langle\psi_{1} \mid \psi_{0}\right\rangle=\int d^{3} r\left[\psi_{0}^{*}\left( \pm i \psi_{0}^{*}\right)-\left(\mp i \psi_{0}\right) \psi_{0}\right]= \pm 2 i \cos 2 \omega_{0} \tau \\
& \left\langle\psi_{0} \mid \psi_{1}\right\rangle+\left\langle\psi_{1} \mid \psi_{0}\right\rangle=\int d^{3} r\left[\psi_{0}^{*}\left( \pm i \psi_{0}^{*}\right)+\left(\mp i \psi_{0}\right) \psi_{0}\right]=\mp 2 \sin 2 \omega_{0} \tau .
\end{aligned}
$$

\section{ENERGY-TIME UNCERTAINITY RELATION}

The total energy of a particle obtained by averaging over the orthogonal periodic temporal interval $T$ in equation 12 is given by,

$$
\omega=\frac{2 \pi}{T}=\frac{E}{\hbar} \Rightarrow T=\frac{h}{E}
$$

Therefore to obtain the total energy of a particle, the terms in equation 8 must be integrated and averaged, over an observed or the measured time period $\Delta T_{\text {measure }}$ which must be an integer multiple of its orthogonal period $T^{\prime}$,

$$
\int_{0}^{\Delta T_{\text {measure }}}\left(\left\langle\psi_{0} \mid \psi_{1}\right\rangle-\left\langle\psi_{1} \mid \psi_{0}\right\rangle\right) d t=0
$$

That is, the observed or the measured time period $\Delta T_{\text {measure }}=n T^{\prime}$ where $n$ is a positive integer, so that the measurement is certain and complete. From equation 18 and 27

$$
\left\langle\psi_{0} \mid \psi_{1}\right\rangle-\left\langle\psi_{1} \mid \psi_{0}\right\rangle= \pm 2 i \cos 2 \omega_{0} \tau= \pm 2 i \cos (2 \omega t-2 \boldsymbol{k} \cdot \boldsymbol{r})
$$

Thus the orthogonality condition in coordinate-time frame given in equation 31 can be obtained when $T^{\prime}=T / 2$ so that the measurement of $E$ is certain and complete.

$$
E=\frac{h}{T}=\frac{n h}{2 \Delta T_{\text {measure }}} \Rightarrow E\left(\Delta T_{\text {measure }}\right)=\frac{n h}{2}
$$

This relation implies that for a given period of measurement $\Delta T_{\text {measure }}$, there exists a minimum energy quantum that can be measured, without any uncertainty, for at least one cycle of its periodic wave $(n=1)$. Further, it shows that the minimum uncertainty of the energy measurement for a given $\Delta T_{\text {measure }}$ time period is,

$$
\begin{gathered}
(\Delta E)_{\min }=\frac{h}{2 \Delta T_{\text {measure }}} \\
(\Delta E)(\Delta T) \geq(\Delta E)_{\min }\left(\Delta T_{\text {measure }}\right) \Rightarrow(\Delta E)(\Delta T) \geq \frac{h}{2} .
\end{gathered}
$$


Equation 34 gives the energy-time uncertainty relation, arising from the complementary wave interpretation. However, as for the interpretation given for uncertainty principles in quantum mechanics, the two observables must arise from two operators which satisfy the canonical-commutation rule. As time $(\Delta T)$ is not considered a quantum mechanical operator, there is some confusion with regards to the energy-time uncertainty relation in quantum mechanics. Nevertheless, in 1945, Mandelshtam and Tamm derived a non-relativistic energytime uncertainty [19] for a quantum system in a stationary state, taking into account the lifetime of the state to change its expectation value, appreciably.

However, the confusion related to time, not being considered a quantum mechanical operator does not arise, since the observables in the energy-time uncertainty relation are related to a set of complementary waves and their period. Further, the complementary wave interpretation shows that the energy-time uncertainty relation is not a statement related to the observer-effect or the measurement-disturbance based on the precision of the measurement but a fundamental statement arising from the complementary nature of momentum-energy and rest-energy waves associated with a particle in motion.

\section{MOMENTUM-POSITION UNCERTAINITY PRINCIPLE}

As $\psi_{0}$ and $\psi_{1}$ are a complementary wave pair, any of the two physical quantities associated with them cannot be measured with arbitrary precision, in accordance with the uncertainty principle. For instance, one cannot know both the position (rest-energy) and the momentum (momentum-energy) of a particle in motion simultaneously, with absolute precision. This we identify as the uncertainty principle formulated by Heisenberg [20]. For two canonically conjugate operators $A, B$ i.e., $[A, B]=i \hbar$, the uncertainties are given by,

$$
\langle\Delta A\rangle\langle\Delta B\rangle \geq \frac{\hbar}{2}
$$

If two operators $\hat{A}_{0}$ and $\hat{A}_{1}$ are associated with a pair of complementary waves $\psi_{0}$ and $\psi_{1}$ respectively, then,

$$
E \psi=\beta m c^{2} \psi_{0}+\alpha^{j} p_{j} c \psi_{1}=\hat{\beta}\left\langle\hat{A}_{0}\right\rangle \psi_{0}+\hat{\alpha}\left\langle\hat{A}_{1}\right\rangle \psi_{1}
$$

Where $\left\langle\hat{A}_{0}\right\rangle$ and $\left\langle\hat{A}_{1}\right\rangle$ represent the expected values of the operators $\hat{A}_{0}$ and $\hat{A}_{1}$ respectively.

$$
\text { Let }|f\rangle=\left|\left(\hat{\beta}\left\langle\hat{A}_{0}\right\rangle-\left\langle\hat{A}_{0}\right\rangle\right) \psi_{0}\right\rangle ; \quad|g\rangle=\left|\left(\hat{\alpha}\left\langle\hat{A}_{1}\right\rangle-\left\langle\hat{A}_{1}\right\rangle\right) \psi_{1}\right\rangle
$$

where $f=\Delta A_{0}=\left(\hat{\beta}\left\langle\hat{A}_{0}\right\rangle-\left\langle\hat{A}_{0}\right\rangle\right) \psi_{0} ; \quad g=\Delta A_{1}=\left(\hat{\alpha}\left\langle\hat{A}_{1}\right\rangle-\left\langle\hat{A}_{1}\right\rangle\right) \psi_{1}$. Then

$$
\left\|\Delta A_{0}\right\|^{2}=\langle f \mid f\rangle ; \quad\left\|\Delta A_{1}\right\|^{2}=\langle g \mid g\rangle .
$$


From Cauchy-Schwartz inequality:

$$
\langle f \mid f\rangle\langle g \mid g\rangle \geq|\langle f \mid g\rangle|^{2} \Rightarrow\left\|\Delta A_{0}\right\|^{2}\left\|\Delta A_{1}\right\|^{2} \geq|\langle f \mid g\rangle|^{2}
$$

$\langle f \mid g\rangle$ in general is a complex number say $c$. Therefore,

$$
|\langle f \mid g\rangle|^{2}=c^{*} c=\frac{1}{4}\left[\left(c+c^{*}\right)^{2}-\left(c-c^{*}\right)^{2}\right]=\frac{1}{4}\left[(\langle f \mid g\rangle+\langle g \mid f\rangle)^{2}-(\langle f \mid g\rangle-\langle g \mid f\rangle)^{2}\right]
$$

$$
\begin{aligned}
& \langle f \mid g\rangle=\left\langle\psi_{0}\left(\hat{\beta}\left\langle\hat{A}_{0}\right\rangle-\left\langle\hat{A}_{0}\right\rangle\right) \mid\left(\hat{\alpha}\left\langle\hat{A}_{1}\right\rangle-\left\langle\hat{A}_{1}\right\rangle\right) \psi_{1}\right\rangle \\
& \langle f \mid g\rangle=\left\langle\psi_{0}\left|\hat{\beta}\left\langle\hat{A}_{0}\right\rangle \hat{\alpha}\left\langle\hat{A}_{1}\right\rangle\right| \psi_{1}\right\rangle+\left\langle\psi_{0}\left|\left\langle\hat{A}_{0}\right\rangle\left\langle\hat{A}_{1}\right\rangle\right| \psi_{1}\right\rangle-\left\langle\psi_{0} \hat{\beta}\left\langle\hat{A}_{0}\right\rangle\left|\left\langle\hat{A}_{1}\right\rangle\right| \psi_{1}\right\rangle-\left\langle\psi_{0}\left\langle\hat{A}_{0}\right\rangle\left|\hat{\alpha}\left\langle\hat{A}_{1}\right\rangle\right| \psi_{1}\right\rangle
\end{aligned}
$$

$$
\begin{aligned}
& \langle f \mid g\rangle=\left\langle\psi_{0}\left|\hat{\beta}\left\langle\hat{A}_{0}\right\rangle \hat{\alpha}\left\langle\hat{A}_{1}\right\rangle\right| \psi_{1}\right\rangle-\left\langle\psi_{0}\left|\left\langle\hat{A}_{0}\right\rangle\left\langle\hat{A}_{1}\right\rangle\right| \psi_{1}\right\rangle . \\
& \langle g \mid f\rangle=\left\langle\psi_{1}\left|\hat{\alpha}\left\langle\hat{A}_{1}\right\rangle \hat{\beta}\left\langle\hat{A}_{0}\right\rangle\right| \psi_{0}\right\rangle-\left\langle\psi_{1}\left|\left\langle\hat{A}_{1}\right\rangle\left\langle\hat{A}_{0}\right\rangle\right| \psi_{0}\right\rangle .
\end{aligned}
$$

Adding equations 41 and 42,

$$
\langle f \mid g\rangle+\langle g \mid f\rangle=\left\langle\left\{\psi_{0} \hat{\beta}\left\langle A_{0}\right\rangle, \hat{\alpha}\left\langle A_{1}\right\rangle \psi_{1}\right\}\right\rangle-\left\langle\left\{\psi_{0}\left\langle A_{0}\right\rangle,\left\langle A_{1}\right\rangle \psi_{1}\right\}\right\rangle .
$$

As there is no linear correlation between $\hat{\beta}\left\langle A_{0}\right\rangle$ and $\hat{\alpha}\left\langle\hat{A}_{1}\right\rangle$ the correlation coefficient between them is zero:

$$
\begin{gathered}
C C_{\beta A_{0} \alpha A_{1}}=\frac{\left\langle\left\{\left(\hat{\beta}\left\langle\hat{A}_{0}\right\rangle-\left\langle\hat{A}_{0}\right\rangle\right) \psi_{0},\left(\hat{\alpha}\left\langle\hat{A}_{1}\right\rangle-\left\langle\hat{A}_{1}\right\rangle\right) \psi_{1}\right\}\right\rangle}{2 \Delta A_{0} \Delta A_{1}}=\frac{\left\langle\left\{\hat{\beta}\left\langle\hat{A}_{0}\right\rangle \psi_{0}, \hat{\alpha}\left\langle\hat{A}_{1}\right\rangle \psi_{1}\right\}\right\rangle-\left\langle\left\{\psi_{0}\left\langle A_{0}\right\rangle,\left\langle A_{1}\right\rangle \psi_{1}\right\}\right\rangle}{2 \Delta A_{0} \Delta A_{1}}=0 \\
\langle f \mid g\rangle+\langle g \mid f\rangle=0 .
\end{gathered}
$$

Therefore equation 40 reads:

$$
\left(\Delta A_{0}\right)\left(\Delta A_{1}\right) \geq \frac{i}{2}(\langle f \mid g\rangle-\langle g \mid f\rangle)=\frac{1}{2}|(\langle f \mid g\rangle-\langle g \mid f\rangle)|
$$

as $(\langle f \mid g\rangle-\langle g \mid f\rangle)$ is complex. Subtracting equation 43 from 42 and using $\alpha \beta=-\beta \alpha$ :

$$
\langle f \mid g\rangle-\langle g \mid f\rangle=\left\langle A_{0}\right\rangle\left\langle A_{1}\right\rangle\langle\hat{\beta} \hat{\alpha}\rangle\left(\left\langle\psi_{0} \mid \psi_{1}\right\rangle+\left\langle\psi_{1} \mid \psi_{0}\right\rangle\right)-\left\langle A_{0}\right\rangle\left\langle A_{1}\right\rangle\left(\left\langle\psi_{0} \mid \psi_{1}\right\rangle-\left\langle\psi_{1} \mid \psi_{0}\right\rangle\right)
$$


From equation 27 and 28,

$$
\begin{gathered}
\langle f \mid g\rangle-\langle g \mid f\rangle=\left\langle A_{0}\right\rangle\left\langle A_{1}\right\rangle\langle\hat{\beta} \hat{\alpha}\rangle\left(\mp 2 \sin 2 \omega_{0} \tau\right)-\left\langle A_{0}\right\rangle\left\langle A_{1}\right\rangle\left( \pm 2 i \cos 2 \omega_{0} \tau\right) \\
|\langle f \mid g\rangle-\langle g \mid f\rangle|^{2}=4\left\langle A_{0}\right\rangle^{2}\left\langle A_{1}\right\rangle^{2} \quad\left(\alpha^{2}=1=\beta^{2}\right) .
\end{gathered}
$$

By setting $\psi_{0}=\psi_{1}=\psi$ in equation (46), the same result represented in equation 47 can be obtained from the Dirac-equation without introducing complementary waves. Equation 47 in terms 45 ,

$$
\left(\Delta A_{0}\right)^{2}\left(\Delta A_{1}\right)^{2} \geq 4\left\langle A_{0}\right\rangle\left\langle A_{1}\right\rangle
$$

Bohr's quantum-rule for angular momentum for an electron is an integer multiple of $\hbar$ interpreted by de Broglie as a standing wave condition. That is the electron, described by a wave and a whole number of wave-lengths must fit along the circumference of the electron's orbit, giving rise to the condition:

$$
r=\frac{n \lambda}{2 \pi}=n \lambda \quad\left(n=\frac{1}{2}, 1, \frac{3}{2}, 2, \ldots\right)
$$

where $n \lambda$ represents half-wave lengths.

Position of a particle is associated with its rest-energy component whereas its momentum is associated with its momentum-energy component. As the two waves $\psi_{0}$ and $\psi_{1}$ are a complementary wave pair, the physical quantities associated with them must be complementary and temporally orthogonal to each other's as well. In the case of an electron, its position (equation 49 ) is associated with $\psi_{0}$ whereas its momentum $(p)$ is associated with $\psi_{1}$.

Therefore,

$$
\hat{A}_{0}\left|\psi_{0}\right\rangle=\left\langle\hat{A}_{0}\right\rangle\left|\psi_{0}\right\rangle=\frac{n}{2 \pi \lambda}\left|\psi_{0}\right\rangle ; \quad \hat{A}_{1}\left|\psi_{1}\right\rangle=\left\langle\hat{A}_{1}\right\rangle\left|\psi_{1}\right\rangle=p\left|\psi_{1}\right\rangle=\frac{h}{\lambda}\left|\psi_{1}\right\rangle
$$

Taking the minimum value of $n=1 / 2$, equation 41 reads:

$$
\left(\Delta A_{0}\right)\left(\Delta A_{1}\right) \geq n \hbar \geq \frac{\hbar}{2}
$$

In the present approach a pair of complementary waves was introduced instead of introducing two operators which satisfy the canonical-commutation rule. Hence the result obtained by using two canonically conjugate operators on a single component wave function and the wave function of a particle in motion associated with a pair of complementary waves are the same. 


\section{TIME ARROW}

The laws of physics that have been articulated from Newton through Maxwell and Einstein, and up until today, show a complete symmetry between past and the future. Nowhere is there any distinction between how the laws look or behave when applied in either direction of time. The laws treat what we call past and future on a completely equal footing. Even though experience reveals over and over again that there is an arrow of how events unfold in time, this arrow is not found in the fundamental laws of physics [21]. In fact, the only law which shows a time direction in contemporary physics is the second law of thermodynamics, where real world phenomena proceed in the direction of increasing entropy. However, all other laws of physics do not seem to require this time arrow.

In the complementary wave interpretation, the complementary wave-pair has a fixed temporal relation between the waves, $\psi_{0}$ and $\psi_{1}$, i.e. one wave always leads the other. Therefore, one can immediately observe that the temporal relation between two complementary waves would exhibit an anti-symmetry, if the time direction is reversed, i.e. if $\psi_{0}$ leads $\psi_{1}$ in the positive time direction, then $\psi_{1}$ would lead $\psi_{0}$ when the time direction is reversed. This we identify as a possible explanation for the existence of a time-arrow, which determines how events unfold in time.

For instance, the non-relativistic hydrogen spectral series is obtained by using the Schrodinger equation, treating the electron in the hydrogen atom as a single-component wave function:

$$
E \psi=-\frac{\hbar^{2}}{2 m} \nabla^{2} \psi-\frac{e^{2}}{4 \pi \varepsilon_{0} r} \psi
$$

This can be alternatively achieved by interpreting the wave function to be associated with a pair of complementary waves, the kinetic-energy component given by the momentum of the particle, associated with the $\psi_{1}$ and the static potential energy component associated with the wave $\psi_{0}$ which are temporally related,

$$
E \psi=-\frac{\hbar^{2}}{2 m} \nabla^{2} \psi_{1}-\frac{e^{2}}{4 \pi \varepsilon_{0} r} \psi_{0}
$$

However, in contrast to the single-component Schrodinger equation, this treatment exhibits an anti-symmetrical temporal relation for the evolution of the hydrogen atom's wave function and dictates a time-arrow.

\section{CONCLUSIONS}

The concept that the wave function of a particle in relativistic motion is associated with a pair of complementary waves was presented interpreting the Dirac equation in terms of complementary waves. Additionally, the two energy components in Einstein's energymomentum equation were shown to arise from the proposed complementary waves which are temporally orthogonal to each other. As the complementary wave pair gives rise to an 
invariant scalar product, the conventional four-vectors were obtained. The energy-time and momentum-position uncertainty relations were derived from the complementary waveinterpretation and they suggest that the observer-effect or the measurement-disturbance is not what determines both the energy-time and momentum-position uncertainty inequalities. That is, even with a set of hypothetical absolute measurements, the two uncertainty relations would hold true, based on the interpretation presented in this paper. Further, in accordance with the uncertainty principle, it suggests that any pair of physical quantities associated with the two complementary waves in a four-vector cannot be known simultaneously, with absolute precision and the motion of a particle is a quantized phenomena. A possible explanation to the time-arrow problem was presented by showing that the proposed complementary wave interpretation dictates an anti-symmetric relation, if the time direction is reversed. By applying the complementary wave-interpretation to the hydrogen atom's wave function, a proof for the existence of its time-arrow was obtained. This may be identified as a possible theoretical explanation to the existence of a time arrow, other than the wellestablished explanation from the second law of thermodynamics.

\section{ACKNOWLEDGMENTS}

The first author is deeply indebted to J. A Gunawardena, whose suggestions and continuous encouragement helped him immensely to investigate and further refine the work presented in this paper.

\section{References}

[1] M. Planck, Annalen der Physik 4 (1901) 553-563.

[2] A. Einstein, Annalen der Physik 17 (1905) 132-148.

[3] P. A. M. Dirac, Proc. Roy. Soc. Lond. A 117 (1928) 610-624.

[4] P. A. M. Dirac, Proc. Roy. Soc. Lond. A 126 (1930) 360-365.

[5] P. A. M. Dirac, Proc. R. Soc. Lond. A 133 (1932) 60-72.

[6] C. D Anderson, Phys. Rev. 43 (1933) 491-494.

[7] E. Majorana, Nuovo Cimento 9 (1932) 335-344.

[8] W. Rarita, J. Schwinger, Phys. Rev. 60 (1941) 61.

[9] A. Proca, J. Phys. Radium 7 (1936) 347-353.

[10] H. J. Bhabha, Rep. Prog. Phys. 10 (1944) 253.

[11] V. Bargmann, E. P. Wigner, PNAS 34 (5) (1948) 211-223.

[12] S. Esposito, Annals of Physics 327(6) (1912) 1617-1644.

[13] E. A. Jeffery, Aust. J. Phys. 31(2) (1978) 137-150.

[14] T. Jaroszewicz, P. S Kurzepa, Annals of Phys. 215 (2) (1992) 457-457.

[15] R. F. Guertin Annals of Phys. 88 (2) (1974) 504-533.

[16] D. Hestenes, J. Math. Phys. 8 (1967) 728-808. 
[17] C. Daviau, Found. Phys. 23 (1993) 1431-1443.

[18] L. de Broglie, Ann. de Physique 3 (1925) 22-128; Am. J. Phys. 40(9) (1972) 1315-1320.

[19] L. I. Mandelshtam, I. E. Tamm, J. Phys. (USSR) 9 (1945) 249-254.

[20] W. Heisenberg, Zeitschrift für Physik 43 (1927) 172-198.

[21] B. Greene, The Fabric of the Cosmos: Space, Time, and the Texture of Reality, Random House, Inc., New York. 2004. 